\title{
Imaging the mouse as a whole
}

\author{
vDISCO combines tissue clearing with nanobody-based immunolabeling for reliable high-contrast imaging of the \\ intact mouse.
}

$\mathrm{C}$ learing methods render opaque tissues and even whole animal bodies transparent. This transparency allows the imaging of fluorescently labeled cells or structures within samples. Yet fluorescent reporters are typically not bright enough to be imaged deep within even a cleared mouse body, necessitating the dissection of the samples. Thus, cleared mice have so far not been analyzed as a whole in any depth or detail.

"For me, the whole motivation of looking into the whole mouse, making it transparent and imaging, is to be able to study the big picture in an unbiased way," says Ali Ertürk from Ludwig-Maximilians University in Munich, Germany. For him, it is important to keep the mouse intact, as many diseases affect the whole body, and it is therefore important to analyze the processes of interest in the context of the intact body. In contrast, standard histological approaches force researchers to focus on individual tissues without providing a comprehensive view.

Ertürk and his team previously developed uDISCO, a clearing method based on organic solvents, which enabled clearing of whole animals such as mice and rats. However, it was not possible to image throughout the whole animals. "You have to use transgenic reporter mice, which usually express fluorescent proteins, and fluorescent proteins are dim compared to bright dyes," explains Ertürk. In addition, the dim signal is further attenuated in thick samples.

To overcome these limitations, the team has now combined the uDISCO approach with high-pressure perfusion to deliver nanobodies uniformly to the whole body. This combined approach is called vDISCO, short for "nanobody $\left(\mathrm{V}_{\mathrm{H}} \mathrm{H}\right)$-boosted $3 \mathrm{D}$ imaging of solvent-cleared organs." Owing to their small size, nanobodies can be easily and uniformly delivered during perfusion. The nanobodies, targeted against reporters such as GFP and RFP, are conjugated to bright Atto dyes. The dyes' emission in the far red spectrum allows their imaging in a spectral window that avoids tissue autofluorescence. The team also successfully used nanobodies against endogenous proteins such as vimentin.

Boosting the endogenous signal with dye-conjugated nanobodies enhanced

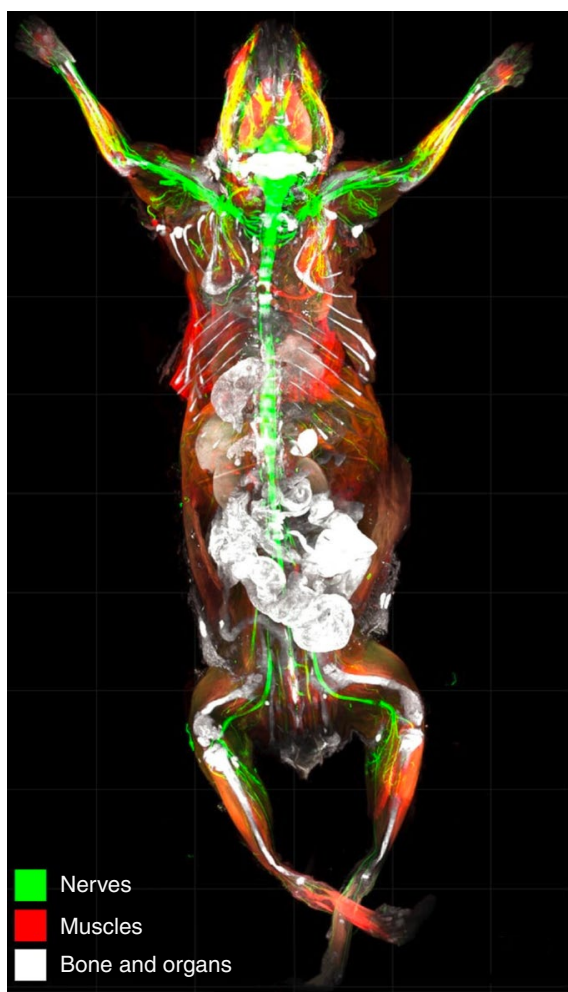

A 3D view of the nerves (green), muscle (red), and other tissue (white) in the mouse. Adapted with permission from Cai et al. (2019), Springer Nature.

the fluorescent signal by two orders of magnitude. The resulting increased contrast then allowed imaging of the cleared animals without further dissection. It is now even possible to "see through the skin because of this 100-plus-times increase of the contrast of the signal," says Ertürk, a feat that had not been attempted previously.

The team verified that the high-pressure perfusion for nanobody delivery does not distort or damage the structural integrity of the samples. For example, the approach allows tracing of complete neurons on the cortex with automated tracing tools.

Ertürk and his team have applied their vDISCO approach in a variety of different contexts. They assessed the effects of traumatic brain injury on peripheral nerve terminals, and observed extensive neurodegeneration in the spinal cord and in the periphery in a mouse model of traumatic brain injury. Furthermore, they took advantage of the fact that vDISCO clears the skull and imaged immune and endothelial cells in the skull and brain. They were able to visualize the short vascular connections between brain surface and skull, which are otherwise difficult to observe, as they are typically destroyed in more conventional preparations. These short vascular connections appear to serve as pathways for immune cells traveling from the skull marrow to the brain after brain injury. Finally, the team used vDISCO to visualize cancer metastasis throughout the whole mouse.

When it comes to clearing methods, "it's very hard to beat vDISCO at this point," says Ertürk. In fact, his team has stopped making further modifications to their clearing protocol. Instead, they are focusing their efforts on the computational aspects. "The next clear step is to do analysis at the wholebody scale in an unbiased way. And that can only happen with deep learning," explains Ertürk. Conventional image analysis tools work well when they are applied to more or less homogeneous samples, but signal contrast and other features vary widely within a whole animal and even within a whole organ such as the brain. Hence, Ertürk and his lab now work on deep learning approaches that could enable them to quantitatively analyze their whole-body data. These quantitative whole-body analyses should provide an unbiased view and a more comprehensive understanding of neuropathologies.

Current light-sheet microscopes lack the capability of imaging whole mice without manual handling of the samples because of their limited field of view and working depth. While clearing of whole animals and quantitative analysis of the large image datasets is now feasible, further developments on the microscopy front would be beneficial.

Nina Vogt

Published online: 27 February 2019 https://doi.org/10.1038/s41592-019-0345-7

Research papers

Cai, R. et al. Panoptic imaging of transparent mice reveals whole-body neuronal projections and skull-meninges connections. Nat. Neurosci. 22, 317-327 (2019). 